Kieler Beiträge zur Filmmusikforschung, 6, 2010 // 37

\title{
Audiovisuelle Brüche: Zur Selbstreflexivität des modernen Films in Chronique d’un été (Chronik eines Sommers, F 1961, Edgar Morin/Jean Rouch) ${ }^{1}$
}

Silke Martin (Weimar)

\section{Zusammenfassung}

Einen wesentlichen Einfluss auf die Ästhetik des cinéma vérité hatte die Entwicklung tontauglicher Handkameras, die den Filmemachern die ästhetische Möglichkeit einer Manipulation und Differenz (im Sinne einer bewussten, eingreifenden Dissoziation und Disjunktion) von Akustischem und Visuellem eröffnete. Anhand ausgewählter Szenen von Chronique D'Un ÉTÉ möchte ich im Folgenden zeigen, wie sich diese Bewegung auf ästhetischer Ebene darstellt. Im Mittelpunkt soll der Akt des Offenlegens und Reflektierens des filmischen Schaffensprozesses stehen, der als wesentlicher Aspekt filmischer Modernität bezeichnet werden kann. ${ }^{2} \mathrm{Zu}$ weiten Teilen folge ich dabei den Schriften von Jean-Louis Comolli und Gilles Deleuze, insbesondere was die prominent beschriebene Durchdringung von dokumentarischem und fiktionalem Stil betrifft, die sich in CHRONIQUE D’uN ÉTÉ nicht nur auf bildlicher, sondern auch und gerade - und das ist das Wesentliche an meiner Argumentation - auf akustischer Ebene nachweisen lässt.

Der Film Chronique D'Un ÉTÉ entstand im Sommer 1960 und ist eine Gemeinschaftsarbeit des Soziologen Edgar Morin und des Ethnologen und Filmemachers Jean Rouch. Mit ChroniQue D'Un ÉTÉ haben Rouch und Morin das Programm des cinéma vérité, einer neuen Strömung des französischen Dokumentarfilms der 1960er Jahre, formuliert. ${ }^{3}$ Die Idee des Films, die Stimmung der Pariser nach dem Algerienkrieg einzufangen, stammt von Morin und stellt ein „soziologisches Experiment“ dar, das lose Interviews auf der Straße ,[...] zu Portraits von konkreten Personen - Arbeitern, Studenten, Lebenskünstlern, Intellektuellen-,

Dieser Beitrag stellt eine Erweiterung eines Vortrags dar, den ich beim 22. Film- und Fernsehwissenschaftliches Kolloquium 2009 in Passau gehalten habe. Vgl. dazu Martin (2009).

2 Der Übergang vom klassischen zum modernen Film, der sich in der Mitte des 20. Jahrhunderts vollzieht, ist insbesondere im Kontext des Gegensatzes von klassisch-narrativem Schema und post-narrativem Kino zu sehen. Der moderne Film, der erste Ausprägungen im italienischen Neorealismus zeigt und von der Nouvelle Vague und dem Neuen Deutschen Film fortgeführt wird, distanziert sich von den narrativen Mustern, die der klassische Film ausgebildet hat, indem er sie variiert und fragmentiert. Das so genannte Autorenkino, das in den 1960er Jahren aufkommt, zielt weniger auf Narration, als vielmehr auf Selbstreflexion, nicht auf die Errichtung einer geschlossenen fiktionalen Welt wie im klassischen (Hollywood-)Film, sondern auf die Bewusstwerdung und -machung seines eigenen medialen Status. Indem der moderne Film die Narration aufbricht, macht er ein Denken über sich selbst, seine Regeln und seine Traditionen möglich. Doch bezieht sich die Modernisierung des Films, die um 1960 einsetzt, nicht nur auf Italien, Frankreich oder Deutschland, sondern auch auf andere europäische und außereuropäische Länder, wie es die zahlreichen Neuen Wellen, etwa die britische und brasilianische, sowie die Neuerungen im japanischen und osteuropäischen Kino zeigen. Mit dem Terminus moderner Film beziehe ich mich demnach auf die Modernisierung des Films in den 1960er Jahren, wobei das Medium Film bereits in den 1920er Jahren eine wesentliche Modernisierungsphase durchlaufen hat (Vgl. dazu Fahle 2008, 1; Vgl. auch Martin 2010).

3 Der Begriff cinéma vérité wurde erstmals 1960 von Morin in einem Bericht über das Festival dei Popoli in Florenz verwendet wurde (vgl. Hohenberger 1988, 227). 


\section{Kieler Beiträge zur Filmmusikforschung, 6, 2010 // 38}

die in ihrer natürlichen Lebenswelt vorgestellt werden $[\ldots]^{“}$ verdichtet und ,,$\left.\ldots\right]$ diese einander nicht unbedingt bekannten Menschen zu Diskussionen zusammentreffen [...]“ (Piechota 2008, 80) lässt. Dabei steht die Frage „Comment vis-tu?“ bzw. „Êtes-vous heureux?“ im Mittelpunkt. ${ }^{4}$ Die Arbeitsweise des Films, die strukturell „den - nicht zuletzt durch die Kamera hervorgerufenen - Reaktionen der handelnden Personen“ (Piechota 2008, 80) folgt, wird dabei von den Filmemachern selbst kommentiert. So kündigt Rouchs Stimme am Anfang des Films an: „Ce film n’a pas été joué par des acteurs, mais vécu par des hommes et des femmes qui ont donné des moments de leur existence à une expérience nouvelle de cinémavérité. “5 Auch am Ende von Chronique D’un ÉTÉ wird die Methode des Films von den Autoren reflektiert, indem sie sich bei einem Spaziergang durch das Musée de l'Homme über die Vorgehensweise und Wirkung des Films unterhalten.

Das Programm des cinéma vérité drückt sich nicht nur in den selbstreflexiven Kommentaren der Filmemacher aus, sondern auch in den Reaktionen der Protagonisten auf die Filmemacher und die Anwesenheit der Kamera. Denn die Filmemacher, die selbst Teil des Gefilmten sind, initiieren und provozieren das, was sie filmen, auch, während sie es und indem sie es filmen. Dabei postulierten sie, sich mit der Methode des cinéma vérité dem Leben annähern und eine Wahrheit zeigen zu wollen, die nur mit dem Film gezeigt werden kann. Die Intension bzw. das „Ziel“ des cinéma vérité ist es also,

[...] die Konflikte zu protokollieren, die durch das Vorhandensein von Kamera und Interviewer hervorgerufen werden. Insofern ist Cinéma Vérité ein "Forschungsprozeß" (Morin) und der Film eine "Film-Enquête", eine "Film-Untersuchung". Der Kamera kommt entsprechend ein Charakter als caméra provocateur zu (Wulff 2007).

Doch dient die Kamera nicht nur als Provokateur und „Katalysator bei der Selbstdarstellung der Beteiligten“, sondern sie ist auch, wie Eva Hohenberger am Beispiel von CHroniQue D'Un ÉTÉ beschreibt, ,[...] ihre wahre raison d'être; ohne sie wären die Leute nicht zusammengekommen und kein Film entstanden“" (Hohenberger 1988, 237). Auch andere Filmemacher wie François Reichenbach, Mario Ruspoli und Chris Marker übernahmen die „Programmatik des cinéma vérité“ (Wulff 2007), wobei der Film Le Joli mai (F 1963, Chris Marker) neben Chronique D’un ÉTÉ als „Klassiker des cinéma vérité“ (Hohenberger 1988, 224) gilt.

Nicht nur das cinéma vérité, sondern auch andere Strömungen des Dokumentarfilms dieser Zeit folgten einer ähnlichen Arbeitsweise. So kam beispielsweise das direct cinema, eine Ende der 1950er Jahre in den USA entwickelte Methode des Dokumentarfilms, oder das free cinema in England auf. Da der Begriff des cinéma vérité auch für die amerikanische Dokumentarfilmbewegung des direct cinema benutzt wird, ein und diesselbe Bezeichnung also synonym für zwei verschiedene Programme verwendet wird, kommt es mitunter

\footnotetext{
„Wie lebst Du?“" bzw. „Sind sie glücklich?“.

„Dieser Film wurde nicht von Schauspielern gespielt, sondern von Männern und Frauen gelebt, die Augenblicke ihres Lebens in den Dienst eines neuen Experiments des Cinéma Vérité stellten."Vgl. die deutsche Untertitelung von ChroniQue D'Un ÉTÉ.
} 


\section{Kieler Beiträge zur Filmmusikforschung, 6, 2010 // 39}

auch, so Hohenberger, zu begrifflichen Verwechslungen innerhalb der verschiedenen Programme (vgl. Hohenberger 1988, 228). Den Unterschied zwischen der Arbeitsweise der amerikanischen und der französischen Dokumentaristen sieht Hohenberger unter anderem in der unterschiedlichen Nutzung der neuen, handlichen Kamera mit Direktton.

Natürlich war diese Entwicklung von den neuen leichten Techniken nicht unabhängig, aber während sie in den USA den Dokumentaristen vorbehalten blieben, die ein vorfilmisches Ereignis ,direkt' aufzeichnen wollten, benutzten französische Dokumentaristen wie Rouch oder Reichenbach sie zur aktiven Teilnahme am Geschehen bis hin zur Initiierung des vorfilmischen Ereignisses (Hohenberger 1988, 227).

Obwohl sich die Selbstreflexivität des direct cinema in einer anderen, vielleicht weniger offensichtlichen Form äußert als die des cinéma vérité, ist beiden eines gemeinsam: Indem der Film sich selbst zur Sprache bringt, wird er nicht nur als Darstellung, sondern auch als Darstellendes, nicht nur als Vermittlung, sondern auch als Vermittelndes wahrnehmbar.

Das cinéma vérité stand, wie Hohenberger schreibt, auch in einem besonderen Verhältnis zur Nouvelle Vague, da diese zur selben Zeit wie das cinéma vérité aufkam und nicht nur einen wesentlichen Einfluss auf die Modernisierung des Films in Frankreich, sondern auch in anderen (außer-)europäischen Ländern hatte.

Das wichtigste Ereignis der französischen Filmkultur jener Zeit war zweifellos die Entwicklung der Nouvelle Vague, aber man kann nicht sagen, daß Rouch ihr Produkt gewesen wäre wie etwa Godard oder Truffaut. Rouch hat sich vielmehr ihr zeitlich vorausgehend in der praktischen Filmarbeit für die Ethnologie entwickelt [...], fügte sich dann jedoch erfolgreich in jenes von der Nouvelle Vague geschaffene, veränderte filmkulturelle Klima ein, in dem nicht nur andere Filme als früher produziert wurden, sondern vor allem auch anders über Filme gesprochen und geschrieben wurde (Hohenberger 1988, 224).

Dabei ist der Begriff des cinéma vérité insbesondere dem Kino Dziga Vertovs und Robert Flahertys verpflichtet, wie bei Morin in Chronique d'un film (1962) nachzulesen ist: „Cinéma-vérité, cela signife que nous avons voulu éliminer la fiction et nous rapprocher de la vie. Cela signife que nous avons voulu nous situer sur une ligne dominée par Flaherty et Dziga Vertov." "Auch Rouch sieht die Vorbilder seines filmischen Werkes in Vertov und Flaherty, über die er schreibt: „Beide waren Filmemacher, die sich mit den Möglichkeiten der Vermittlung von Realität beschäftigten“ (Rouch 1978, 5).

Doch nicht nur Vertov und Flaherty, sondern auch das National Film Board of Canada und der Kameramann Michel Brault hatten maßgeblichen Einfluss auf das Schaffen Rouchs und dessen filmische Auseinandersetzung mit der Realität, vor allem was die technische Entwicklung des Films und die

„Cinéma vérité bezeichnet die Art und Weise, wie wir die Fiktion eliminieren und uns dem Leben annähern wollen. Und, wie wir uns auf einer Linie platzieren wollen, die von Vertov und Flaherty dominiert ist“ (Morin 1962, 41). 
Bewegungsmöglichkeit der Kamera betraf. Rouch schreibt:

Erst die Verwegenheit eines jungen Filmteams vom National Film Board of Canada erlöste die Kamera aus ihrer Unbeweglichkeit [...] Seitdem haben Kameradesigner beträchtliche Anstrengungen unternommen, die Handlichkeit und Ausgewogenheit der Filmkamera zu verbessern. Heute können sich alle 'cinéma-direct'Kameraleute mit ihren Kameras bewegen; sie sind zur lebenden Kamera, zum 'Kino-Auge' Wertows geworden (Rouch 1978, 11; vgl. Hohenberger 1988, 239).

Dabei bezog sich die technische Neuerung, die Rouch anstrebte, nicht nur auf die Kamera, sondern auch auf die Tonapparatur. Denn Rouch wollte neben einer handlichen und leichten Kamera, die einen größeren Bewegungsspielraum bei der Bildaufzeichnung ermöglichte, eine Tontechnik, die es ihm gestattete, den Ton direkt am Drehort aufzunehmen. Die Originaltonaufnahmen sollten die oftmals unpassenden, durch nachträgliche Vertonung hinzugefügten Off-Stimmen ersetzen. In Folge dessen arbeitete Rouch seit 1951 mit Originalton, der $\mathrm{zu}$ jener Zeit noch von einem Tonmann mit einem $30 \mathrm{~kg}$ schweren Tonbandgerät aufgenommen werden musste. Auf akustischer Ebene stellten sich Rouchs Filme fortan als eine Kombination aus „Originalton, Kommentar und einer nachträglichen Kommentierung durch die Gefilmten“ dar, wobei die Tonaufnahme dabei noch sehr beschwerlich war (Hohenberger 1998, 240).

Erst die Entwicklung zweier Erfindungen, die von Brault, Rouch und der Firma Eclair realisiert wurden, verschafften Rouch während der Dreharbeiten von CHRONIQUE D'UN ÉTÉ schließlich die technischen Möglichkeiten, die er sich für sein filmisches Schaffen erhoffte:

\begin{abstract}
Man arbeitete mit dem Prototyp der ersten Synchronkamera, der tragbaren KMT Coutant-Mathot 16-mmKamera mit einem großen Magazin und dem Pilotone-System, das es der Kamera ermöglichte, simultan mit einem Nagra-Rekorder, einem hochempfindlichen Schweizer Tonbandgerät, das den Ton unabhängig von der Bewegung und Entfernung der Kamera aufzeichnen konnte, zu filmen. Michel Brault hatte hierfür erstmals Krawattenmikrofone eingesetzt (Piechota 2008, 78; vgl. Hohenberger 1988, 240 ff).
\end{abstract}

Durch die tragbare und leichte Kameratechnik wurde, wie Piechota schreibt, eine andere Art des Filmens, auf akustischer wie auf visueller Ebene möglich (Piechota 2008, 79). ${ }^{7}$

Auch Comolli führt die Verbreitung der neuen Dokumentarfilmströmung, die er - etwas ungenau - direct nennt, auf die verbesserten technischen Bedingungen jener Zeit zurück: „Die Verbreitung des direct verdankt sich zunächst einem technischen Fortschritt. Das 16mm-Format expandiert und verbessert sich zuerst aufgrund des Amateurfilm-Marktes und dann dank des Fernseh-Marktes“ (Comolli 1969, 259). Zum einen ist es das „anpassungsfähigere“ und „risikofreudigere“ Personal, das die Dreharbeiten erleichtert, zum anderen

\footnotetext{
Wobei CHRONIQUE D'UN ÉTÉ noch nicht über einen durchgehenden Synchronton verfügte. Den ersten vollständigen Synchrontonfilm konnte Rouch erst 1965 drehen, ,[...] mit LA GOUMBÉ DES JEUnes NOCEURS, einem Kurzfilm über eine Tanz- und Musikgruppe in Abidjan“ (Hohenberger 1988, 241).
} 


\section{Kieler Beiträge zur Filmmusikforschung, 6, 2010 // 41}

das empfindlichere Filmmaterial, das die künstliche Beleuchtung zunehmend überflüssig macht. In Folge dessen kann, wie Comolli schreibt, ,überall schnell und unauffällig“ gedreht werden. Die Kameras sind einfacher in ihrer Handhabung, sie werden kleiner, leichter und sie sind, wie er hinzufügt, ,[...] synchron und leise und machen Studioarbeit bzw. Nachsynchronisation überflüssig“ (Comolli 1969, S. 249).

Die neue Art des Filmens, die mit der Einführung tontauglicher Handkameras möglich wurde, äußert sich meines Erachtens insbesondere in einem Auseinanderdriften der Bild- und Tonebene, die eine filmische Selbstreflexion möglich macht. ${ }^{8}$ Interessant ist in diesem Zusammenhang vor allem jene Szene, in der verschiedene Personen in einer Fabrikhalle bei der Arbeit gezeigt werden (ab 00:17:42). Indes ein lauter Geräuschteppich ertönt, der die unterschiedlichen Maschinengeräusche bündelt, fragmentiert und serialisiert die Kamera hingegen einzelne Personen (in überwiegend nahen und halbnahen Einstellungen) und hebt deren Körper, Gesichter und Bewegungen hervor. Es folgt eine zweite Bildserie, die verschiedene Arbeiter beim Essen zeigt, während im Hintergrund weiterhin, allerdings etwas leiser, die Maschinengeräusche zu hören sind. Als die Arbeiter schließlich beim Nachhausegehen gezeigt werden, wird der Geräuschteppich langsam ausgeblendet und Straßenlärm eingeblendet. Obwohl in dieser Szene also nicht nur verschiedene Protagonisten (mehrere Arbeiter), sondern auch verschiedene Tätigkeiten (Arbeiten, Essen, nach Hause gehen) auf bildlicher Ebene gezeigt und bewusst fragmentiert werden, der Film sich durch serielle Bilder und Schnitte somit selbst zur Sprache bringt, bleibt der Ton hingegen derselbe (monotone Maschinengeräusche), wird dabei aber leiser, bis er schließlich ganz verstummt (laut, leise, ausgeblendet) und in einen anderen Ton (Straßenlärm) übergeht. Die Dissoziation von Bild und Ton äußert sich hier in dem Gegensatz von visueller Fragmentierung und akustischer Bündelung, die durch die gleichzeitige Aufnahmemöglichkeit beider Bestandteile wahrnehmbar wird.

Diese Szene macht noch auf eine andere wesentliche Differenz aufmerksam, nämlich auf die unterschiedlichen Aufnahme- und Abspielmodi von Bild und Ton im Film. Denn während das Bild linear und diskontinuierlich aufgenommen wird, wird der Ton hingegen zirkulär und kontinuierlich aufgezeichnet. Bei der Projektion des Films muss dann das diskontinuierliche Abspiel der Bilder mit dem kontinuierlichen Bewegen der Tonspur gekoppelt werden. In der Fragmentierung des Bilderflusses und der Bündelung des Akustischen zeigt sich in dieser Szene auch der Widerspruch, der zwischen Tonspur und Bild bei Aufnahme und Abspiel des Films existiert.

\footnotetext{
Die Überlegungen zu audiovisuellen Brüchen im modernen Film gehen auf Überlegungen zu einer filmischen Evolution des Akustischen zurück, die ich bereits an anderer Stelle entwickelt habe und die sich in aller Kürze folgendermaßen beschreiben lässt: Beruht der klassische Film tendenziell auf einem Zusammenspiel von Bild und Ton im Kontext der Geschichte, so äußert sich der moderne Film eher in einer Trennung von Akustischem und Visuellem. Wird die Asynchronie von Bild und Ton im klassischen Film vermittelt dargestellt, so bricht der moderne Film die narrative Verbindung von Akustischem und Visuellem auf und lässt diese sich unabhängig voneinander entfalten. Nach einer Herausstellung des Akustischen im postmodernen Film führt der Film der Zweiten Moderne in eine Gleichzeitigkeit von Harmonisierung und Dissonanz von Akustischem und Visuellem, so dass die klassische und die moderne Form, Narration und Reflexion miteinander konfrontiert und zusammengeführt werden. Vgl. dazu ausführlich Martin 2010 oder - überblicksartig - Martin 2008.
} 


\section{Kieler Beiträge zur Filmmusikforschung, 6, 2010 // 42}

Doch verweist diese Szene noch auf eine dritte wesentliche Differenz des optischen und des akustischen Raums, nämlich darauf, dass der Raum des Sehens ein geometrischer und logischer Raum ist, der vektoriell gegliedert ist und durch optische Wahrnehmung errechnet wird. Der Raum des Hörens hingegen ist ein topologischer Raum, der unmittelbar und stets in seiner Gesamtheit gegeben ist und in dem alles gleichzeitig zu hören ist (Engell 2004). Indem ChroniQUe D'UN ÉTÉ Bildserien präsentiert, die einzelne Personen und Tätigkeiten fragmentiert, wird die Errechnung des Filmbildes durch die Montage betont und dieser Vorgang, der im Film normalerweise unbemerkt abläuft, bewusst gemacht. Der monotone Geräuschteppich in der Fabrikhalle, der sich aus Überlagerungen aller real erklungenen Geräusche zusammensetzt, hingegen zeigt, dass der Raum des Hörens stets in seiner Gesamtheit gegeben ist und einzelne Töne nicht oder nur sehr schwer isoliert werden können. Um derartige Tonüberlagerungen, die beim Drehen mit Direktton entstehen, zu vermeiden, wird im klassischen Hollywoodfilm die Tonspur nachsynchronisiert. ChroniQUE D'Un ÉTÉ hingegen hebt diesen Aspekt durch die Verwendung von Direktton noch hervor.

Die manipulativen Eingriffe gegen die konventionalisierten Bild-Ton-Beziehungen des klassischen Films zeigen sich auch in jener Szene, in der Marceline über ihre Zeit im Konzentrationslager spricht (ab: 00:53.05). Während Marceline in einem Gespräch mit den anderen Protagonisten erklärt, dass die Tätowierung auf ihrem Arm keine Telefonnummer ist, sondern eine Nummer, die ihr im Konzentrationslager gegeben wurde, schweift die Kamera ab und zeigt ihren Arm, ihr Gesicht und schließlich, nachdem sie zu ihren Gesprächspartnern hinüber geschwenkt hat, ihre Hand, die eine Rose streichelt. Neben dem Abschweifen des Bildes von Marcelines Rede, das so die Kluft zwischen Sichtbarem und Hörbarem im Film wahrnehmbar macht, ist insbesondere die nächste Einstellung interessant, in der Marceline in einer Totalen zu sehen ist, wie sie über die Place de la Concorde läuft und von ihrer Deportation erzählt. Zunächst ist unklar, ob es sich um einen inneren Monolog handelt oder ob sie tatsächlich spricht. Traditionell werden, etwa bei David Bordwell und Kristin Thompson, interne diegetische Töne von externen diegetischen Tönen unterschieden, wobei erstere im Gegensatz zu zweiteren keine physikalische, sondern eine mentale Ursache haben (vgl. Bordwell/Thompson 1993, 310). Indem die Stimme in dieser Szene dem Bild zunächst nicht eindeutig zugeordnet werden kann, da Marcelines Mundbewegung nicht oder nur schemenhaft zu erkennen ist, beginnt der Zuschauer darüber nachzudenken, ob die Figur tatsächlich spricht oder ob sie nur laut denkt. Als Marcelines Gesicht dann in einer Nahaufnahme zu sehen ist, erkennt der Zuschauer, dass es sich um ihre externe diegetische Stimme handeln muss, da sich Marcelines Mund bewegt. Indem zunächst unklar ist, woher die Stimme kommt, wird auf die Existenz von internen und externen diegetischen Stimmen aufmerksam gemacht. Als dann schließlich klar ist, dass es sich bei Marcelines Stimme um eine externe Stimme handelt, wird die Grenze zwischen interner und externer Stimme wieder aufgeweicht, indem der Zuschauer nun realisiert, dass Marceline einen inneren Monolog hält, der geäußert wird. 


\section{Kieler Beiträge zur Filmmusikforschung, 6, 2010 // 43}

Schließlich, nach einem Schnitt, ist Marceline zu sehen, wie sie sich auf einer Straßenkreuzung auf die Kamera zubewegt und in die eindrucksvolle Kulisse der Halles hineinläuft. Aufgrund des starken Gegenlichts ist ihr Gesicht kaum zu erkennen. Indes sie von ihrer Deportation erzählt und auf die Kamera zuläuft, entfernt sich die Kamera dennoch in einer Rückwärtsbewegung von ihr. Marceline wird kleiner und kleiner, während ihre Stimme durch das Krawattenmikrofon und den Nagra-Rekorder, der sich in ihrer Tasche befindet, gut zu hören ist. „Das ,Dekor` der Halles wird so immer mächtiger, während Marceline, die seufzt und wieder seufzt und - „Oh, papa!“ - ihren Vater anruft, am Ende der langen Einstellung nur noch ein kleiner Punkt ist“" (Piechota 2008, 82). Diese Unstimmigkeit zwischen der zunehmend größer werdenden Entfernung der Figur und der gleich bleibenden Lautstärke der Stimme macht den Zuschauer auf eine wichtige Konvention des Tonfilms aufmerksam, die sich nach ersten Experimenten mit dem neuen Medium in den 1930er Jahren etabliert hat und die besagt, dass Dialoge, auch wenn sich die Entfernung der Figur oder die Einstellungsgröße ändert, in einer kontinuierlichen und gut hörbaren Lautstärke wiedergegeben werden müssen, um die Verständlichkeit und Hörbarkeit der Rede zu garantieren. Diese Reflexion klassischer Regeln der Tonverwendung im Film wird durch die Rückwärtsbewegung der Kamera und die so entstehende Distanz von Bild und Ton wahrnehmbar.

Diese Szene stellt sich nicht nur in ästhetischer, sondern auch in inhaltlicher Hinsicht als manipuliert dar. Denn Marceline selbst schätzt ihr Verhalten in dieser eindrucksvollen Szene(rie) als gespielt und inszeniert ein, wenngleich dieses auch, wie sie betont, reale Aspekte ihrer Person enthält (vgl. Rouch/Morin 1962, 165). In diesem Zusammenhang kann das Verhalten von Marceline auf der Place de la Concorde und in Les Halles als „Manipulation der Realität“ bezeichnet werden, bei der sich der Zuschauer fragt,

[...] was Manipulation ist, oder anders, wo die Wahrheit aufhört und die Fiktion beginnt. Das Cinéma-Vérité provoziert und produziert in gewisser Weise also erst das, was es gleichzeitig dokumentiert. Diese „artifizielle“ Form der Wahrheit - und hier liegt die Übereinstimmung mit Vertovs Kinopravda - ist nicht empirisch angelegt, sondern meint, ganz im Gegenteil, eine nur mit den Mitteln des Films herstellbare Wahrheit (Piechota 2008, S. 82).

In derartigen Filmen kommt es, wie diese Szene zeigt, zu einer Durchdringung und Annäherung von Dokument und Fiktion. Diese Annäherung bezieht sich, wie Comolli bereits Ende der 1960er Jahre feststellte, nicht nur auf den Dokumentarfilm, sondern auch auf den Spielfilm:

Im modernen Kino kristallisiert sich zusehends eine bestimmte Tendenz heraus: Der fiktionale Film greift immer deutlicher auf Techniken und Methoden des cinema direct zurück. Ganz sicher in L'Amour fou (Rivette), aber auch in Partner (Bertolucci), La Collectioneuse (Rohmer) und den Filmen von Godard und Gorrel. Genauso sicher in L'enfance Nue (Pialat) und Faces (Cassavetes). An der Grenze zum Paradoxen aber auch in Die Chronik der Anna Magdalena Bach (Straub) und Silence et Cri (Jansco) ... In Ergänzung dazu konstituieren sich Filme, die in erster Linie dem cinema direct zugehören, als Erzählungen und neigen 


\section{Kieler Beiträge zur Filmmusikforschung, 6, 2010 // 44}

teilweise oder ganz der Seite der Fiktion zu - sie produzieren und organisieren Fiktionen. Z.B. Le RÈGnE DU Jour (Perrault), aber auch - fast - alle Filme von Rouch; einige Filme von Warhol, ja selbst, schon damals wiederum an der Grenze zum Paradoxen, La Rosière de Pessac von Eustache und La Rentrée des Usines WONDER (film de mai) (Comolli 1969, 242).

Bei dieser Tendenz, die damals erst im Entstehen war, heute jedoch zu einer gängigen Praxis im Kino geworden ist, „durchdringen“ und „vermischen“ sich die bis dahin weitgehend getrennten und gegensätzlichen Richtungen des „Dokumentarischen“ und des „Fiktionalen“ und treten in einen wechselseitigen „Austauschprozeß“ ein. Die Annäherung von Dokument und Fiktion findet dabei in der Form statt, dass beide ,[...] aufeinander reagieren, einander verändern, einander umformen - und am Ende möglicherweise ununterscheidbar werden“ (Comolli 1969, 242).

Comolli beschreibt diese filmische Strömung als eine „gegenseitige Veränderung von Welt und Film“ (Comolli 1969, S. 257). Denn es existiert keine „vor-filmische Welt“ mehr, auf die sich der Film bezieht und die er darstellt, sondern nurmehr eine ,[...] filmische Welt, die durch den Film und im Film, die simultan und gemeinsam mit der Herstellung des Films erzeugt wird“ (Comolli 1969, 259). Dabei „,...] geht im cinema direct das gefilmte Ereignis dem Film, seinen Dreharbeiten, nicht voraus, sondern wird von ihnen produziert" (Comolli 1969, 265). Die Neuerungen, die durch das cinema direct initiiert werden, beschreibt er folgendermaßen:

In dem Moment, wenn sich der Film durch das direct mit dem Leben nicht mehr über ein System der Reproduktion, sondern der reziproken Produktion verbindet, so daß der Film gleichzeitig (Perrault, Rouch) von den Ereignissen und Situationen produziert wird wie er sie produziert (und aufgrund der Tatsache dieser doppelten Produktion ihrer beider Reflexion und Kritik betreibt), in diesem Moment findet sich der andere (größere) Teil des Films, in dem tausend Definitionen und Widersprüche unentwirrbar verbunden schienen, in einer einzigen Definition gebündelt und auf eine einzige Dimension reduziert: Repräsentation (Comolli 1969, 252).

Dabei infiziert und beeinflusst das cinema direct das Kino der Repräsentation, und zwar in der Art, dass es dieses „modifiziert“ und „überwindet“, so dass eine kategorische Trennung beider Bereiche zunehmend unmöglich wird (Comolli 1969, 253/254). Indem Comolli mit dieser These - sicherlich als einer der ersten den Status des Dokumentarfilms als Gattung in Frage stellt, „kollabiert gleichsam jene stabile Differenz zwischen Welt und Film, die die Vorstellung des Dokumentarfilms als Aufzeichnung oder Abbild erst ermöglicht hat" (Hohenberger 2000, 31). In Folge dessen bezeichnet Hohenberger Comollis Argumentation auch als dekonstruktive Dokumentarfilmtheorie, die nicht mehr mit konventionellen Gattungsbegriffen operiert, sondern - im Gegenteil - mit dem cinéma verité die Trennung von Film und Welt und somit den Begriff des Dokumentarischen als obsolet entlarvt. 
Ebenso, wie sich der Bereich des Dokumentarischen und des Fiktionalen vermischt, durchdringt sich im cinéma vérité aber auch, wie Deleuze schreibt, der Bereich des Subjektiven und des Objektiven. Unter objektiv versteht Deleuze dabei „,...] das, was die Kamera »sieht«, und [unter] subjektiv das, was die Figur sieht“ (Deleuze 1997, 195). Die Kamera sieht und zeigt demnach einerseits den Protagonisten und andererseits das, was der Protagonist sieht. „Man kann also von der Annahme ausgehen, daß die Erzählung die Entwicklung von zwei Arten von Bildern - den objektiven und subjektiven - ist sowie ihr komplexes Verhältnis, das sich [...] in einer Identität [...] auflösen muss“ (Deleuze 1997, 195). Diese Differenzierung und Identifizierung des Subjektiven und des Objektiven wird nun im modernen Film von einer ,anderen Art der Erzählung“, so Deleuze, „in Frage gestellt“. Diese macht es möglich, die Trennung der „objektiven indirekten Erzählung vom Standpunkt der Kamera“ und die „subjektive direkte Erzählung vom Standpunkt der Person“ zugunsten einer „freien indirekten (subjektiven) Rede“ zu unterlaufen (Deleuze 1997, 196). Die Überwindung des Subjektiven und des Objektiven, aus der die freie indirekte Rede hervorgeht, steht dabei, wie Deleuze schreibt, mit einer neuen Form des Sprechaktes, der für das moderne Kino bezeichnend ist, dem Fabulierakt, in Zusammenhang. ${ }^{9}$ Wobei es nicht nur die Filmemacher, sondern auch die Figuren sind, die sich verändern und die die freie indirekte Rede in den Film einführen. Denn die Figur ist, wie Deleuze schreibt, weder „real“ noch fiktiv“

[...] ebenso wie sie nicht mehr objektiv betrachtet wird oder selbst subjektiv betrachtet. Vielmehr handelt es sich um eine Person, die Übergänge und Grenzen überwindet, weil sie als eine reale Person erfindet und umso realer wird, je besser sie erfunden hat (Deleuze 1997, 200).

Aus dieser Perspektive zeigt sich der Akt des Fabulierens nicht nur in der Szene, in der Marceline auf der Place de la Concorde über ihre Zeit im Konzentrationslager spricht, sondern auch in der Schlusssequenz von Chronique D'un ÉTÉ (ab: 01:22.22), in der Morin und Rouch von der Kamera begleitet durch den Saal des Musée de l'Homme spazieren und über das Verhalten der Protagonisten und deren Reaktion auf den Film sprechen (die Protagonisten haben zuvor bei einem Screening Ausschnitte des Films gesehen und im Anschluss daran mit den Filmemachern über den Film gesprochen). Während dieses Gesprächs im Musée de l'Homme beurteilen die Filmemacher das Verhalten von Marceline auf der Place de la Concorde mit folgenden Worten:

9 Deleuze unterscheidet im Tonfilm zwischen drei verschiedenen Arten des Sprechakts, nämlich ,[...] zwischen dialogischen Sprechakten (die sich vornehmlich im »On« und im relativen »Off« abspielen), reflexiven Sprechakten (die vor allem im absoluten Off anzutreffen sind) und schließlich Fabulierakten [...] (jenen geheimnisvollen Akten der »Legendenbildung in flagranti«, die deswegen rein zu nennen sind, weil sie autonom sind und nicht mehr dem visuellen Bild angehören) [...].“ (Deleuze 1997, 323). Wobei Deleuze die zwei Aspekte des off, den relativen und den absoluten, wie folgt beschreibt: verweist das relative off auf ein „nicht sichtbares Ensemble“, das sich im Umfeld des Bildes befindet und jederzeit sichtbar werden kann, so bezeichnet das absolute off, das sich ,außerhalb des homogenen Raums und der homogen Zeit" befindet, hingegen ein „radikaleres Anderswo“, das ,[...] kein Ensemble mehr ist und nicht zum Bereich des Sichtbaren gehört [...].“ (Deleuze 1997, 34). Während also dialogische und reflexive Sprechakte in Verbindung mit dem Visuellen stehen (da sie entweder im on, im relativen oder im absoluten off des Bildes stattfinden), hat sich der Fabulierakt des modernen Films bereits vom visuellen Bild gelöst. 


\section{Kieler Beiträge zur Filmmusikforschung, 6, 2010 // 46}

Morin: .... Nous sommes allés un peu plus avant - Les gens dès qu'ils sont un peu plus sincères que dans la vie, on leur dit, ou bien: vous êtes des cabotins, des comédiens, ou bien on leur dit vous êtes exhibitionistes $[\ldots]$

Rouch: - Eux ne peuvent pas le savoir. Tu comprends lorsque par exemple Marceline dit qu'elle jouait sur la Place de la Concorde - on était témoins? ...

Morin: - Qui ....

Rouch: - ...Elle ne jouait pas!

Morin: - Si elle jouait on peut dire que c'était la partie la plus authentique d'elle-même quand elle parlait de son père - c'est pas un jeu tu comprends, on peut pas appeler ca un jeu ...

Rouch: - Bien sûr.

Morin: - C'est-à-dire que ce film à la différence du cinéma habituel, nous réintroduit dans la vie. Les gens sont devant le film comme dans la vie de tous les jours c'est-à-dire qu'ils ne sont pas guidés, parce que nous n'avons pas guidé le spectateur - nous ne lui avons pas di tun tel est gentil - un tel est méchant - un tel est sympathique - un tel est intelligent, et alors devant ces gens là qu'ils pourraient rencontrer dans la vie ils sont désemparés, ils sentent qu'ils sont mis en cause eux-mêmes, ils se sentent concernés et ils essayent de refuser (vgl. Rouch/Morin 1962, 129). ${ }^{10}$

Interessant ist in dieser Szene vor allem die Selbsteinschätzung der Filmemacher, die, indem sie über die Reaktionen der Figuren sprechen, das Programm des cinéma vérité formulieren. Rouch und Morin beschreiben Chronique D'Un ÉTÉ als einen Film, der ihres Erachtens und im Gegensatz zu herkömmlichen Filmen ins Leben zurückführt und die Authentizität der Figuren zeigt. Sie stellen fest, dass das Verhalten der Figuren nicht erfunden, sondern echt ist (obwohl Marceline selbst ihr Verhalten als gespielt empfindet, sie dies aber, so die Filmemacher, nicht besser wissen kann). Die Filmemacher produzieren und erfinden ihren Film in dem Moment, in dem sie selbst zu Figuren werden und dabei über das sprechen, was die anderen Figuren gesagt haben und was sie als authentisch bezeichnen. Diese Paradoxie und Komplexion von Realität und Fiktion ist im Grunde genommen eine doppelte, da sie sich nicht nur auf Marcelines Rede, sondern auch auf die Rede von Rouch und Morin, die Marcelines Rede reflektiert, bezieht. Eine andere, vielleicht deutlichere Grenzüberschreitung und Paradoxie zeigt sich in der Selbsteinschätzung der Filmemacher, die Figuren nicht gelenkt haben zu haben, nicht gesagt zu haben, wie sich diese verhalten sollen, ob sie böse oder freundlich, sympathisch oder intelligent sein sollen, obwohl es zugleich offensichtlich ist, dass das Verhalten der Figuren durch die Filmemacher und die Anwesenheit der Kamera provoziert wurde.

\footnotetext{
${ }^{10}$ Morin: ... Wir sind ein bisschen weiter gegangen. Wenn sich die Leute etwas ehrlicher zeigen, wirft man ihnen vor: ihr seid Mimen, Komödianten, oder man nennt sie Exhibitionisten [...] Rouch: Sie können es nicht wissen. Als Marceline zum Beispiel sagte, daß sie uns auf der Place de la Concorde etwas vorspiele, waren wir Zeugen, dass sie nicht spielte. Morin: Man kann sagen, daß das die echteste Szene war. Als sie von ihrem Vater erzählte, das war nicht gespielt. Das kann man nicht Spiel nennen. Das heißt, daß dieser Film im Gegensatz zum normalen Kino uns ins Leben zurückführt. Die Leute verhalten sich vor dem Film wie vor ihrem täglichen Leben. Das heißt, sie werden nicht gelenkt. Wir haben ihnen nicht gesagt: Dieser ist freundlich, dieser böse, dieser sympathisch, intelligent ... Vor diesen Leuten also, denen sie im Leben begegnen könnten, fühlen sie sich selber in Frage gestellt, fühlen sich betroffen und sie wehren sich dagegen. Vgl. die deutsche Untertitelung von ChroniQue D'UN ÉTÉ.
} 


\section{Kieler Beiträge zur Filmmusikforschung, 6, 2010 // 47}

Interessant ist in diesem Zusammenhang auch, dass Rouch an anderer Stelle das cinéma vérité als ein „Kino der Lügen“ bezeichnet und somit selbst auf die Paradoxie aufmerksam macht, die dem cinéma vérité innewohnt.

Ich betrachte [...] das cinéma vérité als ein Kino der Lügen, das davon abhängt, wie man sich selbst belügt. Wenn man ein guter Geschichtenerzähler ist, dann ist die Lüge wahrer als die Realität und wenn man ein schlechter Geschichtenerzähler ist, dann ist die Wahrheit schlechter als eine halbe Lüge (Rouch, zit. nach Hohenberger 1988, 231).

Hervorzuheben ist in dieser Szene auch, dass die Filmemacher die Methode des Films reflektieren, während der Film noch nicht zu Ende oder abgeschlossen ist. Auch in der Szene, in der die Figuren in einem Kinosaal über ihren eigenen Film diskutieren, beurteilt sich der Film bereits selbst, ohne sein Ende erreicht zu haben. Indem die Autoren und Figuren innerhalb des Films über den Film nachdenken, wird die Reflexion des Films in den Film selbst verlagert. Zudem werden die Protagonisten bei der Diskussion im Kinosaal in den Prozess des Filmemachens einbezogen, ebenso wie am Anfang des Films, als Rouch und Morin Marceline erklären, sie solle „enthemmt und frei erzählen“, Marceline jedoch entgegnet, dass sie dies nicht könne, weil sie „eingeschüchtert“ sei. Die Filmemacher unterbreiten ihr daraufhin das Angebot, Dinge, die ihr nicht gefallen, aus dem Film herauszuschneiden und beteiligen sie somit am Schaffensprozess des Films, indem sie ihr ein (wenn auch nur begrenztes) Mitspracherecht am Film geben. Die Beteiligung Marcelines an der Entstehung des Films zeigt sich auch in der nächsten Szene, in der sie Passanten an einer Metro-Station fragt „Etes-vous heureux?“" und somit selbst zur Interviewerin wird, nachdem ihr eine ähnliche Frage („Comment vis-tu?“) von Rouch und Morin gestellt wurde. Die Beteiligung der Figuren am Schaffensprozess des Films wird dann wiederum reflektiert, indem diese Gegenstand der Rede von Rouch und Morin ist.

Chronique D'UN ÉTÉ verfügt demnach nicht nur über verschiedene Ebenen des Fiktionalisierens, Dokumentierens, Betrachtens und Reflektierens, die sich auf vielfältige Weise durchdringen und vermischen, sondern auch über Autoren, die zu Figuren werden, und Figuren, die zu Autoren werden, wobei beide, Figuren wie Autoren, das reflektieren, was sie im Fabulieren erfinden und was zugleich und paradoxerweise auch echt ist. Demnach sind die Personen weder real noch fiktiv, sondern beides zugleich. (Für mich stellt sich aber vor allem heraus, dass die Frage danach, was fiktiv und was real ist, offensichtlich weit weniger wichtig ist, als bis dahin angenommen.) Es ist auch keine eindeutige Zuordnung des Subjektiven und Objektiven mehr erkennbar, da die Figuren und Autoren ihre Rolle tauschen und in Folge dessen, um eine Wendung von Deleuze aufzugreifen, keine Differenzierung mehr zwischen einer „objektiven indirekten Erzählung vom Standpunkt der Kamera“/des Autors und einer „subjektiven direkten Erzählung vom Standpunkt der Person“ möglich ist (Deleuze 1997, S. 196). 
Kieler Beiträge zur Filmmusikforschung, 6, 2010 // 48

Während dieses Nachdenkens über sich selbst in der Schlusssequenz des Films folgt die Kamera den Filmemachern, umwandert und umkreist sie, sucht ihre Nähe, bis sie sich schließlich abrupt zurückzieht und in der Mitte des Raums stehen bleibt. Während Morin und Rouch in die Tiefe des Bildes laufen und die Figuren sich immer weiter entfernen, bleibt die Lautstärke ihres Gesprächs hingegen gleich. Sind Bild und Ton in dieser Szene zunächst ihrer gegenseitigen Abstimmung verpflichtet, indem die Kamera den Redenden ebenso nahe ist wie das Tonaufnahmegerät der Stimme (es handelt sich hierbei um Krawattenmikrofone, die eine gleichbleibend laute Aufnahme der Stimme gewährleisten), so eröffnen sie schließlich abrupt und offensichtlich eine Kluft zwischen dem, was zu sehen ist, und dem, was zu hören ist.

Dies zeigt, dass in CHRONIQUE D'UN ÉTÉ nicht nur auf inhaltlicher, sondern auch auf ästhetischer Ebene ein Akt des Reflektierens und Offenlegens des filmischen Entstehungsprozesses abläuft. In der Kluft von Sichtbarem und Hörbarem, von Figuren, die sich entfernen, und Stimmen, die bleiben, zeigt sich die bewusste Dissoziation von Bild und Ton, die erst, so meine Schlussfolgerung, durch die gleichzeitige Aufnahmemöglichkeit beider Bestandteile denkbar wurde. Denn für eine derartige Ästhetik war neben der Mobilität der Handkamera insbesondere der Direktton entscheidend, der solche Experimente überhaupt erst möglich machte. Diese neue Art des Filmens, die mit dem cinéma vérité und ähnlichen Strömungen aufkam und die den modernen Film entscheidend geprägt hat, äußert sich, wie sich resümierend feststellen lässt, in einem veränderten Verhältnis von Akustischem und Visuellem. Der Akt des Reflektierens, der sich in CHronique D'Un ÉTÉ vollzieht und der ein Nachdenken des Films über sich selbst möglich macht, kann dabei als ein wesentlicher Aspekt filmischer Modernität beschrieben werden. 


\section{Literatur}

Bordwell, David/Thompson, Kristin (1993) Film Art: An Introduction. New York [u.a.]: McGraw-Hill.

Comolli, Jean-Louis (2000) Der Umweg über das direct (1969). In: Bilder des Wirklichen, Texte zur Theorie des Dokumentarfilms. Hrsg. v. Eva Hohenberger. Berlin: Verlag Vorwerk 8, S. 242-265.

Deleuze, Gilles (1997) Das Zeit-Bild. Kino 2. Frankfurt/Main: Suhrkamp.

Engell, Lorenz (2004) Bild und Ort des Klangs. Musik als Reflexion auf die Medialität des Films. Vortrag, Wissenschaftliches Kolloquium, Filmmusik - Theoriebildung und Vermittlung, Weimar.

Fahle, Oliver (2008) Der Film der Zweiten Moderne oder Filmtheorie nach Deleuze. unveröffentlichter Vortrag, Bochum.

Hohenberger, Eva (1988) Die Wirklichkeit des Films: Dokumentarfilm, ethnographischer Film, Jean Rouch. Hildesheim [u.a.]: Olms.

Hohenberger, Eva (2000) Dokumentarfilmtheorie. In: Bilder des Wirklichen, Texte zur Theorie des Dokumentarfilms. Hrsg. v. Eva Hohenberger. Berlin: Verlag Vorwerk 8, S. 8-34.

Martin, Silke (2008) Vom klassischen Film zur Zweiten Moderne - Überlegungen zur Differenz von Bild und Ton im Film. In: Kieler Beiträge zur Filmmusikforschung 2, Online-Zeitschrift, URL: http://www.filmmusik.uni-kiel.de/kielerbeitraege2/KB2-Martin.pdf

Martin, Silke (2009) Die Manipulation und Differenz von Bild und Ton im cinéma vérité - Chronique D'uN ÉTÉ (Chronik eines Sommers, 1961). In Klimczak, Peter / Großmann, Stephanie (Hrsg.): Dokumentation des 22. Film- und Fernsehwissenschaftlichen Kolloqiums in Passau, Marburg: Schüren Verlag.

Martin, Silke (2010) Die Sichtbarkeit des Tons im Film. Akustische Modernisierungen des Films seit den 1920er Jahren. Marburg: Schüren (im Erscheinen).

Morin, Edgar (1962) Chronique d'un film. In: CHRONIQUE D'UN ÉTÉ, Hrsg. v. Jean Rouch und Edgar Morin. Paris: Inter Spectacles, S. 5-43.

Piechota, Antje (2008) Jean Rouch. Innovationen im Spannungsfeld von Ethnologie und Kino. Saarbrücken: VDM Verlag.

Rouch, Jean/Morin, Edgar (1962) CHRONIQUE D'UN ÉTÉ, Paris: Inter Spectacles.

Rouch, Jean (1978) Die Kamera und der Mensch. In: Freunde der Deutschen Kinemathek 56, S. 2-23.

Vertov, Dziga (1973) Kinoki-Umsturz. In: Dziga Vertov. Schriften zum Film. Hrsg. v. Wolfgang Beilenhoff. München: Hanser, S. 11-25.

Vertov, Dziga (1973) Kinopravda. In: Dziga Vertov. Schriften zum Film. Hrsg. v. Wolfgang Beilenhoff. München: Hanser, S. 103-108.

Wulff, Hans J. (2007) Cinéma Vérité, Stand 140/2007. In: Lexikon der Filmbegriffe, Hrsg. v. Theo Bender und Hans J. Wulff, online: http://www.lexikon.bender-verlag.de (Stand: 12.01.2009). 
Kieler Beiträge zur Filmmusikforschung, 6, 2010 // 50

\section{Empfohlene Zitierweise:}

Martin, Silke: Audiovisuelle Brüche: Zur Selbstreflexivität des modernen Films in Chronique d'un été (Chronik eines Sommers, F 1961, Edgar Morin/Jean Rouch).

In: Kieler Beiträge zur Filmmusikforschung 6, 2010.

URL: http://www.filmmusik.uni-kiel.de/beitraege.htm

Datum des Zugriffs: 1.11.2010.

Kieler Beiträge für Filmmusikforschung (ISSN 1866-4768)

Copyright $\mathbb{C}$ by Silke Martin. All rights reserved.

Copyright (C) für diese Ausgabe by Kieler Gesellschaft für Filmmusikforschung. All rights reserved.

This work may be copied for non-profit educational use if proper credit is given to the author and „Kieler Beiträge für Filmmusikforschung“" 